\title{
Recurrent Acute Necrotizing Encephalopathy in a Canadian Aboriginal Child
}

\author{
Essam Howayyer, Aizeddin A. Mhanni, Jens Wrogemann, Michael S. Salman
}

Can. J. Neurol. Sci. 2011; 38: 925-928

Acute necrotizing encephalopathy (ANE) in childhood was originally described in the Far East. ${ }^{1,2}$ Disease onset is commonly preceded by a viral illness., ${ }^{1,3}$ Neurological deterioration occurs abruptly and rapidly. Neuroimaging is characteristic. ${ }^{4}$ Steroids and less commonly intravenous immunoglobulins have been used with variable outcomes. ${ }^{5}$ More recently, cases have been reported from Europe and North America. ${ }^{3,6,7}$ Here we present the first report of recurrent ANE in a Canadian Aboriginal child.

\section{CASE RePORT}

A previously healthy 34-month-old aboriginal Canadian boy from northern Manitoba presented with a four day history of cough and fever, and two day history of headache and irritability. He was sleepy the day prior to his presentation. There was no history of trauma, travel, rash or toxic ingestion. He did not have seizures. He was initially seen at the local hospital. His Glasgow Coma Scale was six. He was intubated, ventilated, and transferred to the Pediatric Intensive Care Unit at our hospital where he was started empirically on intravenous Cefotaxime, Vancomycin and Acyclovir. His past medical history is unremarkable. The mother was well during pregnancy. He was born at thirty six weeks gestation following an uneventful pregnancy and spontaneous vaginal delivery. His birth weight was $2.7 \mathrm{~kg}$. His development was normal prior to his presentation. The parents are of Canadian Aboriginal Cree descent and are nonconsanguineous.

Serial investigations were done including, full blood count (CBC), electrolytes, ammonia, glucose, liver function tests, cortisol, ACTH, TSH, T3, T4, vitamin $\mathrm{B}_{12}$, lead, antithyroid antibodies, ANCA, ANA, toxicology screen, capillary lactic acid, plasma amino acids, plasma very long chain fatty acids, whole blood palmitate oxidation assay (a rapid screening test for fatty acids oxidation defects), free and total serum carnitine levels, acylcarnitine profile, lysosomal enzymes assay panel, blood cultures; urine amino and organic acids; cerebrospinal fluid (CSF) glucose, protein, cell count, lactic acid, amino acids, oligoclonal bands, bacterial, fungal, and viral cultures including human herpes viruses (lumbar puncture was done twice, four days apart); and stool for Enteroviruses. The results were all normal except for mild elevation of CSF protein on the first lumbar puncture at $0.52 \mathrm{~g} / \mathrm{L}$ (normal range: $0.15-0.4$ ). The results of the oligoclonal bands were negative; however, there was a systemic immune reaction associated with CSF abnormality.

The electroencephalogram (EEG) showed slowing in background activity consistent with an encephalopathic process.
There was also occasional epileptiform activity over the left more than the right hemisphere, especially over the temporal regions. His magnetic resonance imaging (MRI) was abnormal with multiple areas of symmetric non-enhancing increased T2 signal intensity with diffusion restriction. These areas involved the posterior thalami, extreme and external capsules, medial temporal lobes, hypothalamus, midbrain, pons, tail of the caudate nucleus (Figure 1). Spinal MRI showed a focal area of increased T2 signal intensity, with no enhancement or expansion of the spinal cord located on the right side of the spinal cord at C6 and C7 region, and another area extending from mid T11 to mid $\mathrm{T} 12$ region that was associated with some expansion of the cord (Figure 2). A repeat brain MRI four days later, showed some improvement with residual abnormalities in the external and extreme capsule and claustrum bilaterally, hypothalamus, lateral pons, amygdala, and parts of the left frontal lobe. Diffusion weighted images demonstrated edema in these areas but no diffusion restriction, as was seen in the earlier MRI. A provisional diagnosis of ANE was made.

He was treated with a single infusion of intravenous immunoglobulins $(\mathrm{Ig})(1 \mathrm{~g} / \mathrm{kg})$ on the third day of his admission and was extubated two days later. The following day he had improvement in his level of consciousness and started talking. He was discharged home two weeks later. On follow-up two months later, he appeared attentive. His language was delayed for age. He could no longer make three-word sentences but could put two words together. His vocabulary was less than before. His lower limb examination revealed increased tone. There was no weakness or ataxia. Achilles tendons were tight bilaterally. He could walk only with support with a typical spastic diplegic gait. The rest of his exam including his upper limbs was normal.

A developmental assessment at 37 months-of-age revealed global developmental delay in the 18-21 month range. A repeat MRI three months after presentation showed almost complete resolution of all of the previous acute abnormalities, but there was atrophy in some of the previously affected areas (Figure 3).

From the Department of Pediatrics and Child Health (EH, AAM, MSS), Department of Biochemistry and Medical Genetics (AAM), Department of Radiology (JW), Section of Pediatric Neurology (MSS), Faculty of Medicine (EH, AAM, JW, MSS), University of Manitoba, Winnipeg, Manitoba, Canada.

Received March 23, 2011. Final Revisions Submitted May 3, 2011. Correspondence to: Michael S. Salman, Section of Pediatric Neurology, Children's Hospital, AE 308, 820 Sherbrook Street, Winnipeg, Manitoba, R3A 1R9, Canada. 


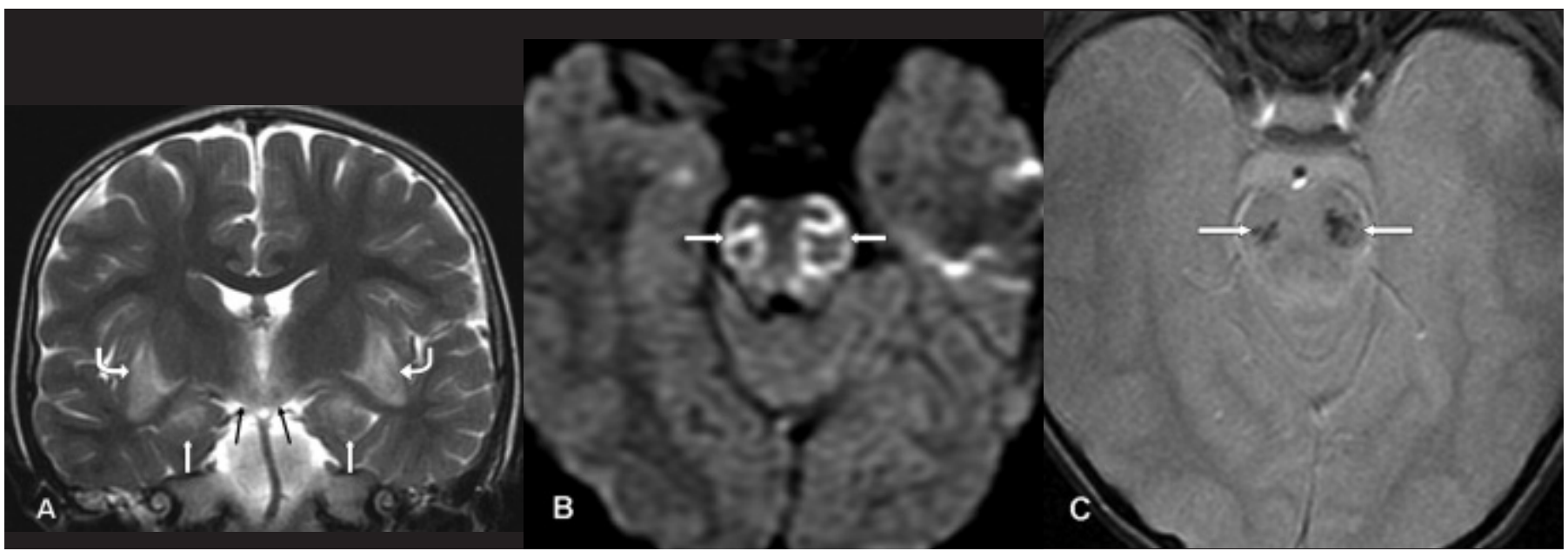

Figure 1: Brain MRI during the initial presentation. A) Coronal T2 weighted image (TR 2950.0, TE 108.1) at the level of foramina of Munroe showing abnormal signal in the external capsule, claustrum and extreme capsule (curved white arrows), hypothalamus (black arrows) and medial temporal lobes (straight white arrows). B) Axial diffusion weighted image (TR 8000.0 TE 83.7) through the pons showing restricted diffusion (arrows). C) Axial gradient echo image (TR 467.0 TE 15.0) through the pons showing hemorrhage (arrows).

One year later, he could make three-word sentences and his gait was only mildly abnormal with no spasticity. At the age of four years and eight months, he made four-word sentences, understood two-step commands, scribbled a circle, jumped, hopped, and climbed up and down the stairs with help. He ran awkwardly but without falling.

He presented again at the age of five years with a one day history of cough and fever followed by decreased level of consciousness. He did not have seizures. He was intubated and ventilated for nine days. He needed a gastrostomy tube due to swallowing difficulties. He received a three days course of methylprenisolone followed by a tapering dose of dexamethasone over a three weeks period. He was started on coenzyme Q10 and L-carnitine supplements. Repeat investigations including CBC, INR, APTT, electrolytes, calcium, glucose, liver function tests, cortisol, ACTH, TSH, T3, T4, vitamin $\mathrm{B}_{12}, \mathrm{C} 3, \mathrm{C} 4, \mathrm{RF}, \mathrm{pANCA}, \mathrm{cANCA}, \operatorname{IgG}, \operatorname{IgM}$, proteinase 3 and myeloperoxidase antibodies, whole blood palmitate oxidation assay, acylcarnitine profile, CLN1 and CLN2 assays and toxicology screen were all unremarkable. Vitamin E was slightly low at $8(12-21 \mu \mathrm{mol} / \mathrm{L})$. The total serum carnitine was low at $20 \mu \mathrm{mol} / \mathrm{L}$ (normal $37-89 \mu \mathrm{mol} /$ ) and the free serum carnitine was low at $17 \mu \mathrm{mol} / \mathrm{L}$ (normal 28-69 $\mu \mathrm{mol} / \mathrm{L}$ ). MELAS, MERRF and NARP point mutation analysis was negative. CSF glucose, protein, cell count, lactic acid were normal. Influenza B was cultured from a nasopharyngeal aspirate. Endotracheal tube grew Morexella cataralis. Bacterial cultures, herpes simplex virus, polymerase chain reaction, and mycoplasma serology were negative. His repeat brain MRI showed new heterogeneous high T2 signal intensity with volume expansion involving the entire pons and midbrain and extending into cerebral peduncles and brachium bilaterally. There was evidence of extensive micro-hemorrhages in the pons. Restricted diffusion in the pons and lower midbrain was seen with heterogenous enhancement of the pons. There was bilateral high $\mathrm{T} 2$ signal intensity without hemorrhage in the region of the

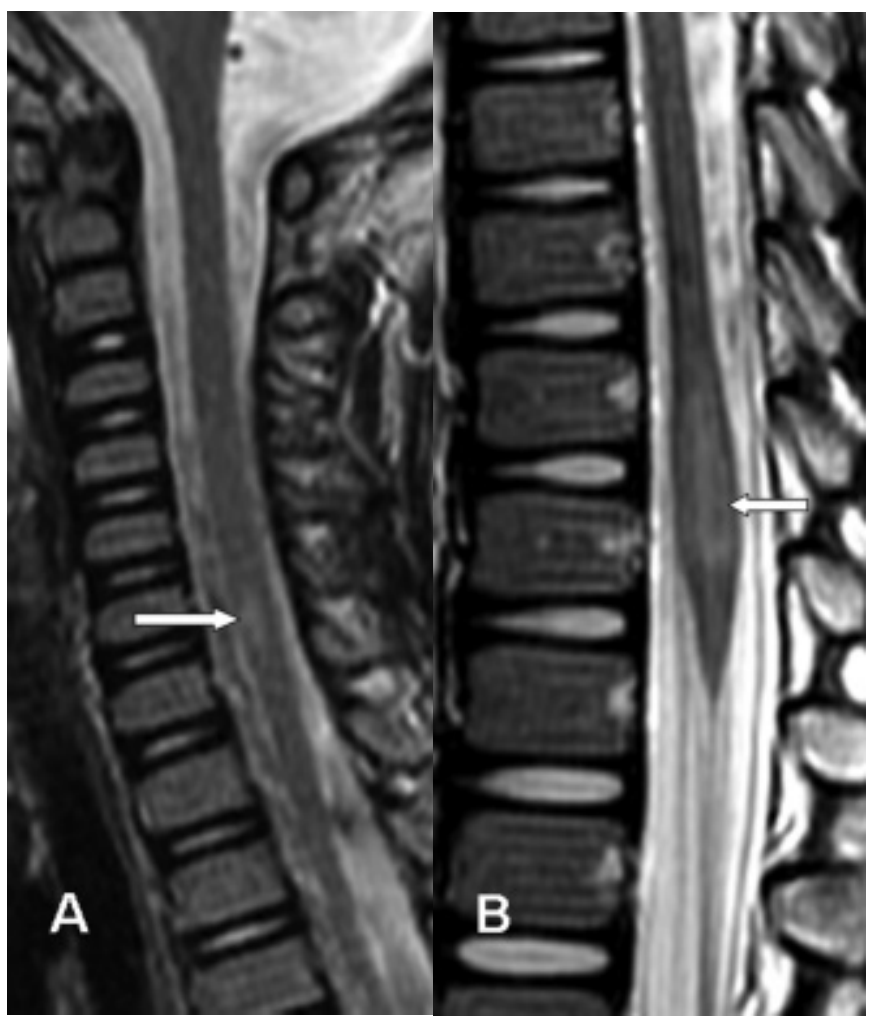

Figure 2: Spinal MRI during the initial presentation. A) Sagittal T2 weighted image (TR 3100.0 TE 112.9) through the cervical spine showing focal high signal behind C6-7 (arrow). B) Sagittal T2 weighted image (TR 3000.0 TE 112.9) through the thoraco-lumbar junction showing high signal and expansion of cord behind T11 and T12 (arrow). 


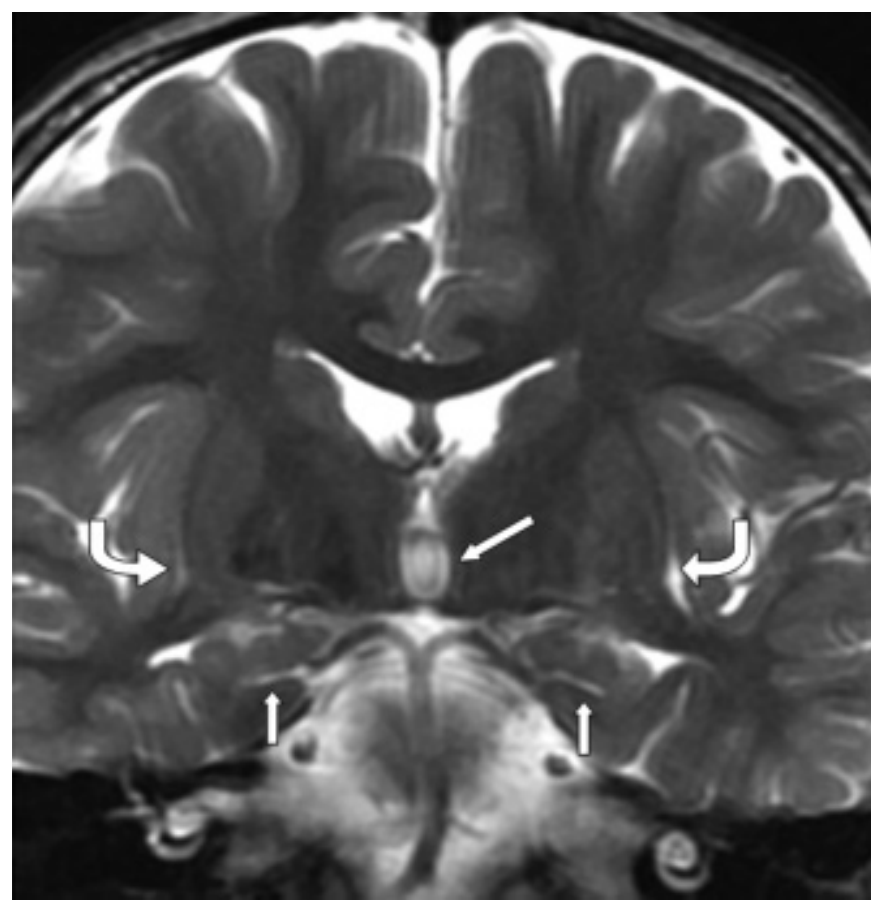

Figure 3: Coronal T2 weighted MRI (TR 3050.0 TE 115.0) at the level of foramina of Munroe three months after the initial presentation showing volume loss in the external capsule, claustrum and extreme capsule (curved white arrows), medial temporal lobes (white arrows) and hypothalamus resulting in ex-vacuo dilatation of third ventricle (oblique arrow).

external and the extreme capsules and posterior limbs of the internal capsule bilaterally. The thalami were normal. Magnetic resonance spectroscopy of the basal ganglia was normal.

A muscle biopsy for routine histology, modified GomoriTrichrome stain and other studies including immunohistochemistry and electron microscopy showed non-specific changes in glycogen content and scattered small fibers but no evidence of mitochondrial diseases. Respiratory chain enzyme analyses in muscle mitochondria revealed mildly reduced activity of all complexes but there was no evident deficiency of any specific complexes. A clinical diagnosis of recurrent ANE was made and mutation analysis of the RANBP2 gene (Molecular Genetics Laboratory, Cincinnati Children's Hospital) revealed that the child is heterozygous for a recently reported disease causing mutation, g.33868A $>$ G substitution. ${ }^{7}$

On follow-up three months later, his developmental milestones had regressed in all domains in comparison to his baseline at the last follow-up after the first episode. He was able to crawl and pull to stand but could not walk. He used a wheelchair. He attempted to scribble but had poor fine motor skills. He was non-verbal but could communicate with his mother using gestures such as head nodding for "yes" or "no". He was fed mostly through his gastrostomy tube. His extraocular movements were full horizontally and vertically. His smooth ocular pursuit was saccadic. He had right exotropia. Bilateral internuclear ophthalmoplegia was present affecting the left more than the right side. His ocular fundus examination was normal.
He had bilateral and mainly lower facial weakness. He had generalized hypertonia especially in the lower limbs and more on the left side. His power was reduced especially in the lower limbs. He could lift both his arms above his head. His right arm and hand strength was nearly normal. His reflexes were brisk and Babinski's sign was positive bilaterally.

\section{Discussion}

Acute necrotizing encephalopathy as a distinct form of acute encephalopathy was described first by Mizuguchi et al. in 1995 . $^{1}$ It was reported predominantly in East Asia, mainly Japan and Taiwan. ${ }^{2}$ However, it has also been reported in non-Asian Caucasian patients outside these countries including North America in Caucasian and non-Caucasian patients. ${ }^{3,6,7}$ Of the few cases reported in Canada, two were Canadian-born children of European ancestry. ${ }^{6}$ In one Canadian report, ethnicity was not mentioned. ${ }^{3}$ Our case expands the ethnic spectrum of this disease. Patients with ANE are usually previously healthy and younger than five years of age. They present with non-specific symptoms associated with fever in most cases, then their neurological status deteriorates abruptly and, in many cases, seizures develop. Our case is consistent with such a presentation; albeit, seizures did not occur. The differential diagnosis of ANE includes Leigh's syndrome, Wernickes encephalopathy, and Rye's syndrome. ${ }^{2}$ The clinical course and the results of the investigations in our patient were not consistent with these disorders. ${ }^{2}$ In ANE, laboratory investigations are nonspecific. Many patients have elevated serum aminotransferase. The CSF protein tends to be high but CSF cell count is normal. ${ }^{1,2}$ Neuroimaging in our patient was characteristic of ANE and led us to the diagnosis. However, we found additional spinal cord abnormalities on MRI, which has been described only recently. ${ }^{7}$ Characteristic brain lesions in ANE can be seen relatively early in the course of the disease both on CT and MRI of the brain. The abnormalities are multiple and symmetrical. ${ }^{1}$ They mainly involve the thalami, brain stem tegmentum, cerebral periventricular white matter and cerebellar medulla. ${ }^{2,8}$ During the acute phase, edematous lesions occur causing necrosis of the involved neurons and glial cells. ${ }^{8}$ Hemorrhage and atrophy can occur. ${ }^{4}$ The apparent diffusion coefficient (ADC) values on diffusion MRI is reported to be high in the center of lesions involving the thalamus or the deep cerebral white matter, suggesting necrosis. ${ }^{8}$ The ADC values at the periphery of these lesions are low, which suggests cytotoxic edema involving neuroglial cells and possibly swelling of myelin. ${ }^{8}$ In addition, very high ADC values have been reported in superficial lesions located outside the thalamus, for example the peripheral portions of the pontine tegmenta. This likely represent extravasations around the arteries and suggests vasogenic edema. ${ }^{8}$ Several viruses have been isolated in patients with ANE. The most common is influenza A and B., ${ }^{1,3}$ We did not find any specific etiology in our patient during the first episode, but Influenza B might have been the trigger for the second episode.

Generally, ANE is considered a monophasic disease. However, recurrence has been reported. ${ }^{7}$ Genetic factors have been recently implicated in the etiology of familial or recurrent

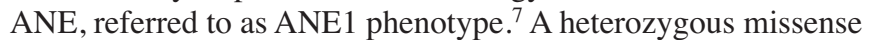
mutation in one of the genes encoding the nuclear pore protein Ran Binding Protein 2 (RANBP2) was identified. This mutation 
occurred in seventy five percent of the familial or recurrent cases while it was not found in those who had an isolated attack of ANE. The inheritance pattern is thought to be autosomal dominant with incomplete penetrance. ${ }^{7}$ Our patient presentation is consistent with ANE1 phenotype where serum transaminases are not typically elevated and MRI shows involvement of additional structures including the external capsule, claustrum, medial temporal lobes, and spinal cord. ${ }^{7}$ The management of ANE is mainly supportive with seizure control, ventilator support, and maintaining the hemodynamic status of the patient. Steroids, both as pulse therapy and as a single dose, and more recently intravenous immunoglobulins have been used. ${ }^{5}$ Steroids used in the first 24 hours of the illness may be associated with better outcome if the lesions do not involve the brainstem. ${ }^{5}$ There is no information available about the best treatment option in recurrent cases. Our patient recovered with mild-to-moderate residual disabilities following the first episode of ANE. However, severe developmental regression followed ANE recurrence. Prognosis in the earlier studies was poor with death or significant neurological sequelae in $65 \%$ of affected patients. ${ }^{2}$ In the cases with recurrence, the outcome is variable, from complete recovery to residual motor and cognitive deficits.

\section{REFERENCES}

1. Mizuguchi M, Abe J, Mikkaichi K, et al. Acute necrotizing encephalopathy of childhood: a new syndrome presenting with multifocal, symmetric brain lesions. J Neurol Neurosurg Psychiatry. 1995;58:555-61.

2. Mizuguchi M. Acute necrotizing encephalopathy of childhood: a novel form of acute encephalopathy prevalent in Japan and Taiwan. Brain Dev. 1997;19(2):81-92.

3. Sazgar M, Robinson JL, Chan AKJ, Sinclair DB. Influenza B acute necrotizing encephalopathy: a case report and literature review. Pediatr Neurol. 2003;28(5):396-9.

4. Kim JH, Kim IO, Lim MK, et al. Acute necrotizing encephalopathy in Korean infants and children: imaging findings and diverse clinical outcome. Korean J Radiol. 2004;5(3):171-7.

5. Okumura A, Mizuguchi M, Kidokoro H, et al. Outcome of acute necrotizing encephalopathy in relation to treatment with corticosteroids and gammaglobulin. Brain Dev. 2009;31(3): 221-7.

6. Kirton A, Busche K, Ross C, Wirrell E. Acute necrotizing encephalopathy in caucasian children: two cases and review of the literature. J Child Neurol. 2005;20(6):527-32.

7. Neilson DE, Adams MD, Orr CM, et al. Infection-triggered familial or recurrent cases of acute necrotizing encephalopathy caused by mutations in a component of the nuclear pore, RANBP2. Am J Hum Genet. 2009;84(1):44-51.

8. Albayram S, Bilgi Z, Selcuk H, et al. Diffusion-weighted MR imaging findings of acute necrotizing encephalopathy. Am J Neuroradiol. 2004;25(5):792-7. 